\title{
Practical waveform inversion in anisotropic media: the natural combination of the data and image objectives
}

Tariq Alkhalifah and Zedong Wu, KAUST

\section{SUMMARY}

Addressing anisotropy in full wavenumber inversion (FWI) is crucial to obtaining credible models, and it is extremely challenging considering the multi parameter nature of the inversion. A successful FWI in anisotropic media takes into account the sensitivity of the data (or the wave) to the long and short wavelength components of the anisotropic parameters. Considering the low sensitivity of FWI to the anellipticity parameter $\eta$ when parametrizing the acoustic transversely isotropic model with the horizontal velocity, $\eta$ and $\epsilon$, we develop a combined FWI and reflection waveform inversion (RWI) to invert for the anisotropic parameters that influence surface seismic data. This practical waveform inversion (PWI) separates the parameters to their resolvable scales, with information accessed from the data fitting (FWI) and the image focusing (RWI) objectives. With this parametrization, the RWI role is to obtain a smooth $\eta$ model, as well as velocity, while FWI focusses on the scattering potential of the horizontal velocity. The parameter $\epsilon$ is used to produce the Born scattered wavefield for the RWI part and eventually fit the amplitude for the imperfect physics in the FWI part.

\section{INTRODUCTION}

Taking anisotropy into consideration in full waveform inversion (FWI) is crucial to obtaining credible models of the Earth (Burridge et al., 1998; Plessix and Cao, 2011; Prieux et al., 2011; Operto et al., 2013). The influence of anisotropy is even more profound on the transmission component of the wavefield from the source, or from a reflector, which is usually constrained and analyzed using Migration velocity analysis (MVA) methods. Alternatively, we can fit the data with a Born scattered wavefield from a predicted perturbation (migration/demigration); we refer to this MVA-like method, as we are evaluating the credibility of the predicted perturbation (Chavent and Plessix, 1999; Clement et al., 2001; Xu et al., 2012), as reflection waveform inversion (RWI). However, anisotropic inversion in general faces many obstacles, the biggest is the multi parameter nature of the inversion. Finding the minimum number of parameters that explain our data at different scales is necessary for a successful waveform inversion. This minimum parameter sensitivity can be attained by choosing the appropriate parameterization for the RWI and FWI parts of the inversion. We consider a transversely isotropic model with a vertical symmetry axis (VTI). Thanks to gravity, such a model is the source of most of the anisotropy we encounter in wave propagation in sub- surface seismic exploration. Inverting for the right parameter combination at the parameters resolvable scale alleviates the Null space that even the full Hessian will not be able to properly address if the update is not linear, like the one proposed here. Thus, the value of the parametrization and identifying its influence is not only important in mitigating the tradeoff, but more so in devising a model that actually influences the data.

For anisotropic media, the short and long wavelength components of the anisotropic parameters have varying influences on the recorded data. Using a representation of the VTI model given by the horizontal velocity $v_{h}$, the anelipticity parameter $\eta$, and our connection to the vertical velocity, $\epsilon$, Alkhalifah (2016) pointed out that we can isolate the $\eta$ influence on the data to mainly its long wavelength component. Conversely, we can isolate the $\epsilon$ influence to its short wavelength component. Thus, we need only to invert for two parameters at each scale. Alkhalifah and Wu (2016) combined FWI and RWI into a single objective functional that naturally adheres to both objectives. Thus, in this abstract, we invert simultaneously for $v_{h}, \epsilon$, and $\eta$ using the new objective function. We set the sensitivity to $\eta$ of the modeled data from the source to zero, and allow $\eta$ to be extracted only from RWI. This will reduce the tradeoff.

\section{THE INVERTIBLE PARAMETERS}

As we combine FWI with RWI, considering an acoustic VTI model, and data acquired on the Earth surface, we must evaluate the scattering potential of FWI (scattering component) and RWI (transmission component). For reflections we contend with those corresponding to horizontal reflectors, where the dipping events influence can be extrapolated as mainly a rotation of the radiation patterns for the horizontal reflector case (Alkhalifah and Plessix, 2014). For transmissions, we keep the scattering angle, $\theta$, open at 180 degrees, and thus, the radiation patterns represent the propagation angle, $\phi$, of the transmission waves. For RWI, such transmission takes place from a secondary source at the reflector, with the depth of this predicted reflector controlled by the background (vertical) velocity. Since this secondary source depends mainly on the vertical velocity, we scale the sensitivity of the data by such dependency, or in other words, we replace the vertical axis with one that keeps the secondary source stationary (i.e. vertical time) to properly include the velocity-depth ambiguity in the sensitivity (radiation) patterns.

Starting from the VTI acoustic wave equations presented by Duveneck et al. (2008), Zhou et al. (2006) showed that they can be written in terms of a pressure field, $p$ 


\section{FWI+MVA+anisotropy}

and its anisotropic perturbation, $q$,

$$
\left\{\begin{array}{l}
-\frac{1}{v_{n}^{2} \rho} \omega^{2} p-\partial_{x}\left(\frac{1}{\rho} \partial_{x}(p+q)\right)-\partial_{y}\left(\frac{1}{\rho} \partial_{y}(p+q)\right) \\
-\frac{1}{\sqrt{1+2 \delta}} \partial_{z} \frac{1}{\rho} \partial_{z} \frac{1}{\sqrt{1+2 \delta}} p=s(\omega) ; \\
-\frac{1}{v_{n}^{2} \rho} \omega^{2} q-2 \eta\left(\partial_{x}\left(\frac{1}{\rho} \partial_{x}(p+q)\right)+\partial_{y}\left(\frac{1}{\rho} \partial_{y}(p+q)\right)\right)=0,
\end{array}\right.
$$

where $s$ is the source term, $\omega$ is the angular frequency, and $\rho$ is the density. However, in this study, following Alkhalifah and Plessix (2014), we consider density constant as $\epsilon($ or $\delta)$ can absorb its role.

The location of the secondary source (governed by the imaging process in RWI) depends on the background velocity used in imaging. Since we place our receivers on the flat Earth surface, the main variation in the source location with respect to the recording plane is vertical. In VTI media, such vertical variation is governed by the vertical velocity. So to keep the source depth stationary, we scale the vertical axis by the vertical velocity equivalent to Alkhalifah et al. (2001) and Plessix (2013), of course implemented there for other reasons. As a result, stating equation 1 in terms of the horizontal velocity combination in the vertically scaled domain, we obtain

$$
\left\{\begin{array}{l}
-\frac{1}{v_{h}^{2}} \omega^{2} p-\frac{1}{1+2 \eta}\left(\partial_{x x}(p+q)-\partial_{y y}(p+q)\right)-\frac{1}{v_{h}^{2}} \partial_{\tilde{z} z} p=s \\
-\frac{1}{v_{h}^{2}} \omega^{2} q-2 \frac{\eta}{1+2 \eta}\left(\partial_{x x}(p+q)+\partial_{y y}(p+q)\right)=0
\end{array}\right.
$$

where for simplicity we stick with the same notations for the wavefields, parameters, and coordinates in the new domain. To remind our selves that the vertical axis is scaled we use $\tilde{z}$ (vertical time) to represent the new vertically-stationary secondary-source axis.

We use equation (1) to develop the radiation patterns for reflection energy considering plane waves (corresponding to state and adjoint state wavefields) approaching the perturbation point from fixed sources and receivers. The details of the derivation is provided by Alkhalifah and Plessix (2014), and the resulting radiation patterns considering perturbations in $v_{h}, \epsilon$, and $\eta$ are shown in Figure 1a. On the other hand, for the transmission radiation patterns from an image controlled source, we utilize equation 2 , with a similar derivation to that of Alkhalifah and Plessix (2014), but we extract here the transmission components. Thus, considering perturbations in the VTI model given by

$$
v_{h}=v_{0}\left(1+r_{v_{h}}\right) ; \quad \eta=\eta_{0}+r_{\eta} ; \quad \epsilon=\epsilon_{0}+r_{\varepsilon},
$$

the resulting scattered wavefield satisfies:

$p_{1}\left(\mathbf{x}_{s}, \mathbf{x}_{r}, \omega\right)=-\omega^{2} s(\omega) \int d \tilde{\mathbf{x}} A\left(\mathbf{x}_{s}, \tilde{\mathbf{x}}, \mathbf{x}_{r}, \omega\right) \mathbf{a}(\tilde{\mathbf{x}}) \cdot \mathbf{r}(\tilde{\mathbf{x}})$

with $A$ as an amplitude function that includes the wave spreading components, among other terms, and

$$
\mathbf{r}=\left(\begin{array}{l}
r_{v_{h}} \\
r_{\eta} \\
r_{\varepsilon}
\end{array}\right) ; \quad \mathbf{a}=\left(\begin{array}{c}
2 \sin ^{2}(\phi) \\
-2 \sin ^{2}(\phi) \cos ^{2}(\phi) \\
0
\end{array}\right)
$$

The coefficients of a define the transmission radiation pattern of each parameter for the given parameterization, $v_{h}, \eta, \epsilon$, with $\phi$ as the transmission angle measured from vertical. Figure $1 \mathrm{~b}$ shows the transmission radiation patterns for the combination used here. The radiation patterns for the $v_{n}, \eta$, and $\delta$ combination, shown in Figure 1c, is derived through a simple linearized relation between the parameters (Alkhalifah and Plessix, 2014). In both cases, the secondary source is given by a $v_{h}$ perturbation extracted from Figure 1a. However, its vertical placement is dependent on the vertical velocity, and thus, as a result of the depth ambiguity, we end up with zero sensitivity of the parameters vertically for the RWI transmissions. In other words, the zero-offset demigrated data from a horizontal reflector produces data equivalent in phase to the observed data regardless of the background velocity (insensitive to velocity). As a result, moveout away from zero offset is required to acquire information to update the background. A similar observation is realized with the differential semblance operator (DSO), as the energy at zero-offset is nearly zero (the repercussion of the annihilator). Also, as expected, we see from Figure 1b, the data is, to the first order, insensitive to $\epsilon$. A similar observation for $\delta$ is realized from Figure 1c. For transmission from a reflector (scatterer), Figure 1b shows that the influence of $v_{h}$ and $\eta$ start at the same scattering angle away from vertical, inducing some tradeoff. On the other hand, with the $v_{n}$ parametrization, the influence of $v_{n}$ and $\eta$ are slightly decoupled as we increase the scattering angle (or offset). Thus, the $v_{n}$ combination might be a better choice for the RWI part of the inversion. If the scatterer corresponds to a perturbation in $\epsilon$ (obtained from the FWI part), we will attenuate the influence of large scattering angles (or large offsets) in RWI, which is desirable when the background velocity is inaccurate, as we will see below.

\section{THE COMBINED INVERSION}

Using the combined FWI and RWI objective functional introduced by Alkhalifah and Wu (2016), we suggest the following modification to include the anisotropy parameters based on the representation, $v_{h}, \eta$, and $\epsilon$ for VTI media:

$$
E_{b}\left(v_{h}, \delta \epsilon, \eta\right)=\frac{1}{2}\left|d_{o}-d_{s}\left(v_{h}\right)-d_{m}\left(v_{h}, \eta, \delta \epsilon\right)\right|^{2} .
$$

The observed data is given by $d_{o}$, while the data, $d_{s}$, correspond to the modeled wavefield, $u_{s}\left(\mathbf{x}_{\mathbf{r}}\right)$, from a source located at $\mathbf{x}_{\mathbf{s}}$, and the data, $d_{m}$, correspond to the modeled wavefield, $\delta u_{s}\left(\mathbf{x}_{\mathbf{r}}\right)$, from a perterbation, all data at receiver positions, $\mathbf{x}_{\mathbf{r}}$. Using this formulation, $\eta$ is weakly invertible from $d_{s}$ (or the FWI part), and $\epsilon$ is weakly invertible from $d_{m}$ (or the RWI part). However, to attenuate the role of far offsets in RWI, we represent the perturbation, as suggested earlier, by $\epsilon(\delta \epsilon)$. The main weakness of the conventional RWI formulation is the requirement that $d_{m}$ matches $d_{o}$ (at least in phase) 


\section{FWI+MVA+anisotropy}

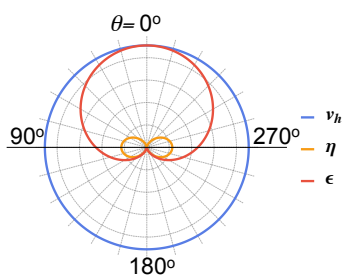

(a)

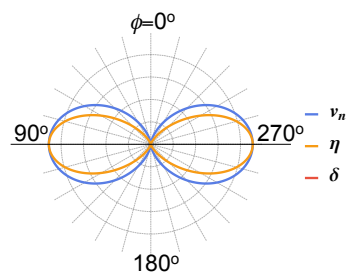

(c)

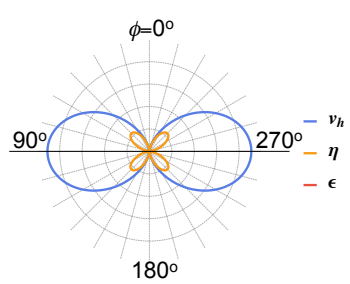

(b)

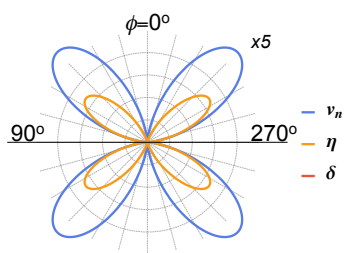

$180^{\circ}$

(d)
Figure 1: Radiation patterns for the parameterization $\left(v_{h}, \eta, \varepsilon\right)$ corresponding to a) a reflection from a horizontal reflector, b) a transmission from scattering at a perturbation in $v_{h}, \mathrm{c}$ ) a transmission from scattering at a perturbation in $v_{h}$ for a parametrization of $\left(v_{n}, \eta, \delta\right)$, and c) a transmission from scattering at a perturbation in $\varepsilon$ for a parametrization of $\left(v_{n}, \eta, \delta\right)$ enhanced by a factor of 5 . The angle $\theta$ is the scattering angle, and $\phi$ is the transmission angle measured from vertical.

at zero offset (Alkhalifah and Wu, 2016). With an inaccurate velocity, this matching is achieved by fitting the near offset part. Thus, there are three advantages in using $\epsilon$ (thanks to its radiation pattern) in the perturbation instead of $\delta v_{h}$. For one, we can include $\delta \epsilon$ to help fit the amplitude (or phase) in RWI. In addition, the Born perturbed wavefield with a perturbation in $\epsilon$ favors fitting the Born scattered data at near offset, a feature needed for a successful RWI, when starting with a poor initial model. The use of $\delta \epsilon$ also allows us to isolate the diving waves to the FWI part. That might help convergence as diving waves provide background updates.

The corresponding wavefields, $u_{s}=\{p, q\}$ and $\delta u_{s}=$ $\{\delta p, \delta q\}$ satisfy the following equations:

$$
\begin{aligned}
L\left(v_{h}, \eta, \epsilon=\eta\right) u_{s} & =s, \\
L\left(v_{h}, \eta, \epsilon=\eta\right) \delta u_{s} & =-\delta L(\delta \epsilon) u_{s}(\mathbf{x}),
\end{aligned}
$$

The wave equation operator, $L$, is given by the Helmholtz form shown in equations 1 and 2 , and thus, $\delta L$ is its perturbation with regard to $\delta \epsilon$. In the operator $L$, we will drop the $\epsilon$ dependency, for simplicity, as we have equated it to $\eta$. Using the extended Lagrangian formulation:

$$
E\left(v_{h}, \eta, \delta \epsilon, u, \delta u, \lambda_{1}, \lambda_{2}\right)=E_{b}-A_{1}-A_{2},
$$

where

$$
\begin{aligned}
A_{1}\left(v_{h}, u, \lambda_{2}\right) & =<\lambda_{2}, L u-s> \\
A_{2}\left(v_{h}, \eta, \delta \epsilon, u, \delta u, \lambda_{1}\right) & =<\lambda_{1}, L \delta u-\delta L u_{s}>
\end{aligned}
$$

where $<$. $>$ stands for inner product over space coordinates. Thus, we evaluate the adjoint-state formulas obtained by setting $\frac{\partial E}{\partial u}=\frac{\partial E}{\partial \delta u}=0$,

$$
\begin{aligned}
& L^{T} \lambda_{1}=-\Delta d\left(\mathbf{x}_{\mathbf{r}}\right) \delta\left(\mathbf{x}-\mathbf{x}_{\mathbf{r}}\right) \\
& L^{T} \lambda_{2}=\delta L \lambda_{1}+L^{T} \lambda_{1}
\end{aligned}
$$

where $\Delta d=d_{o}-d_{s}\left(v_{h}\right)-d_{m}\left(v_{h}, \eta, \delta \epsilon\right)$. Thus, we can define $\lambda_{2}^{\prime}=\lambda_{2}-\lambda_{1}$, and as a result

$$
\begin{aligned}
L^{T} \lambda_{1} & =-\Delta d\left(\mathbf{x}_{\mathbf{r}}\right) \delta\left(\mathbf{x}-\mathbf{x}_{\mathbf{r}}\right), \\
L^{T} \lambda_{2}^{\prime} & =\delta L \lambda_{1},
\end{aligned}
$$

which represents the adjoint wavefields. Finally, taking the derivative of $E$ with respect to the model parameters, where the state and adjoint state variables are considered fixed, we obtain the gradients

$$
\begin{aligned}
\nabla_{v_{h}} E_{b} & =-\frac{\partial L}{\partial v_{h}}\left(u \lambda_{1}+u \lambda_{2}^{\prime}+\delta u \lambda_{1}\right) \\
\nabla_{\eta} E_{b} & =-\frac{\partial L}{\partial \eta}\left(\delta u \lambda_{1}+u \lambda_{2}^{\prime}\right) \\
\nabla_{\delta \epsilon} E_{b} & =-\frac{\partial \delta L}{\partial \delta \epsilon} u \lambda_{1}
\end{aligned}
$$

\section{A NUMERICAL TEST}

We extract a small piece of the Sigsbee model as shown in Figure 2a, label it as the horizontal velocity, and add to it an $\eta$ Gaussian anomaly model shown in Figure $2 \mathrm{~b}$ to represent the true VTI model. The parameter $\epsilon$ is assumed to equal $\eta$. The data are generated from 32 equally spaced shots on the top surface of the model, and receivers are located at every grid point on the surface, with a model grid spacing of 15 meters in each direction. This assumed observed data corresponds to a single frequency of $7 \mathrm{~Hz}$.

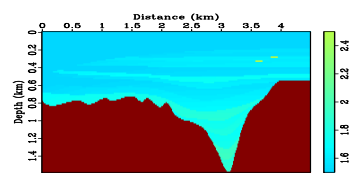

(a)

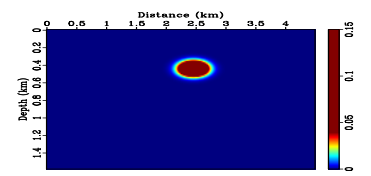

(b)
Figure 2: (a) The exact horizontal velocity extracted from the Sigsbee model. (b) The exact $\eta=\epsilon$, which is a Gaussian anomaly.

We start the inversion with a linearly increasing velocity model between the water bottom and the salt body shown in Figure $3 \mathrm{a}$ and $\eta=0$. We do not update the model in the water layer or in the Salt body (both considered isotropic) throughout the inversion. We apply a 


\section{FWI+MVA+anisotropy}

scattering angle filter (Alkhalifah, 2015) on the updates of both $v_{h}$ and $\eta$, using the efficient scattering enrichment method of $\mathrm{Wu}$ and Alkhalifah (2015). We relax the scattering angle filter only for $v_{h}$ as we expect no reliable high resolution information from $\eta$ (Alkhalifah, 2016). Figure $3 \mathrm{~b}$ shows the initial $\delta \epsilon$ used to generate the scattered wavefield for the RWI part, and a reflects the monofrequency nature of the implementation. We use an LBFGS method to optimize $\delta \epsilon$ at every iteration. On the other hand, a Guass-Newton approach is used to update $v_{h}$ and $\eta$. We apply 4 stages of scattering angle filtering with 20 iterations at each stage. The resulting inverted $v_{h}$ and $\eta$ are shown in Figures $4 \mathrm{a}$ and $4 \mathrm{~b}$, respectively. As expected, we obtain a reasonably high resolution $v_{h}$, shown in the vertical profile in Figure 5a (purple),. We also obtain a smooth $\eta$, both comply well with the true model. The $\eta$ represents a smooth version of the true one, as it corresponds to energy extracted from large scattering angles. The inverted $\delta \epsilon$ shown in Figure $4 \mathrm{c}$ has generally lower energy than the one corresponding to the initial model (Figure 3b) reflecting a better fit to the data. For comparison, we show the isotropic inversion result using the same method and number of iterations (Figure 4d), and from the vertical profile shown in Figure 5a (green) we notice the larger errors brought about from ignoring $\eta$ (the anisotropy).

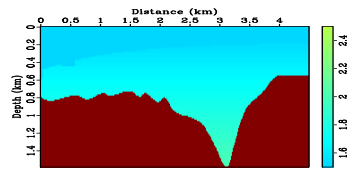

(a)

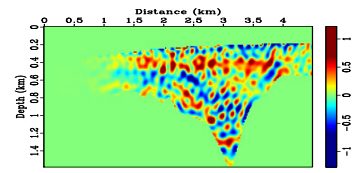

(b)
Figure 3: (a) The initial horizontal velocity for the inversion. (b) The corresponding inverted $\delta \epsilon$.

\section{CONCLUSIONS}

We propose using the natural combination of RWI and FWI in a single objective function to invert for key anisotropic parameters at their resolvable scales. Using a representation of the VTI model given by the horizontal velocity $\left(v_{h}\right), \eta$, and $\epsilon$, we can invert for the long wavelength scale of the $v_{h}$ using the FWI part and inverting for the long wavelength part of $\eta$ as well using RWI. The high resolution $v_{h}$ and $\epsilon$ are obtain from the FWI of reflections as the inversion naturally moves to that part when the models start to induce scattering.

\section{ACKNOWLEDGMENTS}

We thank KAUST for its support. We appreciate the Seismic wave analysis group (SWAG) for a collaborative environment.

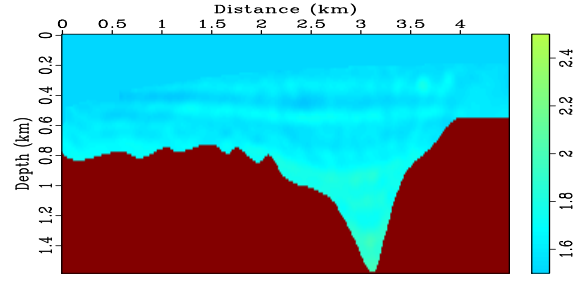

(a)

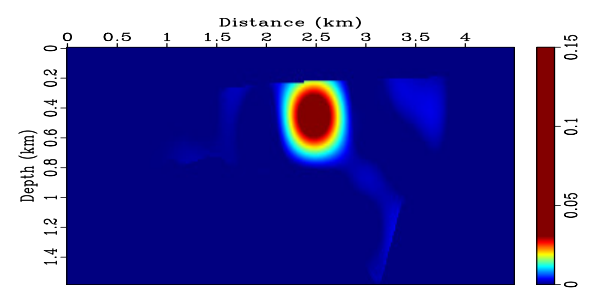

(b)

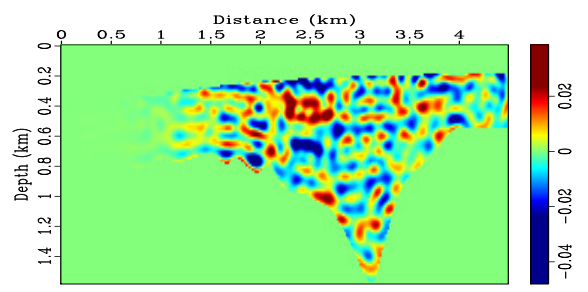

(c)

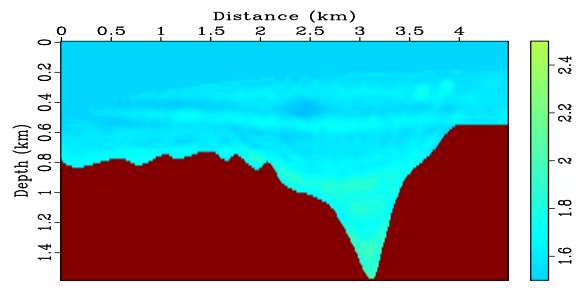

(d)

Figure 4: a) The inverted horizontal velocity. b) The inverted $\eta=\epsilon$. d) The inverted $\delta \epsilon$ corresponding to the inverted model. d) The inverted velocity using an isotropic inversion.

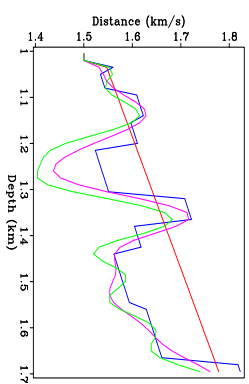

(a)

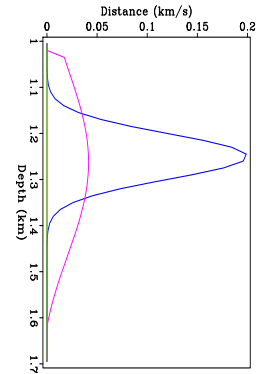

(b)
Figure 5: A vertical profile from location $3.2 \mathrm{~km}$ of the true model (blue), initial model (red), inverted model using the anisotropic assumption (purple), and the inverted model using the isotropic assumption (green) for a) velocity, and b) $\eta$. 
EDITED REFERENCES

Note: This reference list is a copyedited version of the reference list submitted by the author. Reference lists for the 2016 SEG Technical Program Expanded Abstracts have been copyedited so that references provided with the online metadata for each paper will achieve a high degree of linking to cited sources that appear on the Web.

\section{REFERENCES}

Alkhalifah, T., 2015, Conditioning the full-waveform inversion gradient to welcome anisotropy: Geophysics, 80, no. 3, R111-R122, http://dx.doi.org/10.1190/geo2014-0390.1.

Alkhalifah, T., 2016, Research note: Insights into the data dependency on anisotropy: An inversion prospective: Geophysical Prospecting, 64, 505-513, http://dx.doi.org/10.1111/1365-2478.12345.

Alkhalifah, T., and R. Plessix, 2014, A recipe for practical full-waveform inversion in anisotropic media: An analytical parameter resolution study: Geophysics, 79, no. 3, R91-R101, http://dx.doi.org/10.1190/geo2013-0366.1.

Alkhalifah, T., and Z. Wu, 2016, The natural combination of full and image-based waveform inversion: Geophysical Prospecting, 64, 19-30, http://dx.doi.org/10.1111/1365-2478.12264.

Alkhalifah, T., S. Fomel, and B. Biondi, 2001, The space-time domain: Theory and modelling for anisotropic media: Geophysical Journal International, 144, 105-113, http://dx.doi.org/10.1046/j.1365-246x.2001.00300.x.

Burridge, R., M. V. de Hoop, D. Miller, and C. Spencer, 1998, Multi-parameter inversion in anisotropic elastic media: Geophysical Journal International, 134, 757-777.

Chavent, G., and R. Plessix, 1999, An optimal true-amplitude least-squares prestack depth-migration operator: Geophysics, 64, 508-515, http://dx.doi.org/10.1190/1.1444557.

Clement, F., G. Chavent, and S. Gómez, 2001, Migration based traveltime waveform inversion of 2-D simple structures: A synthetic example: Geophysics, 66, 845-860, http://dx.doi.org/10.1190/1.1444974.

Duveneck, E., P. Milcik, P. Bakker, and C. Perkins, 2008, Acoustic VTI wave equations and their application for anisotropic reverse time migration: 78th Annual International Meeting, SEG, Expanded Abstracts, 2186-2190, http://dx.doi.org/10.1190/1.3059320.

Operto, S., Y. Gholami, V. Prieux, A. Ribodetti, R. Brossier, L. Metivier, and J. Virieux, 2013, A guided tour of multi-parameter full-waveform inversion with multi-component data: From theory to practice: The Leading Edge, 32, 1040-1054, http://dx.doi.org/10.1190/tle32091040.1.

Plessix, R., 2013, A pseudo-time formulation for acoustic full waveform inversion: Geophysical Journal International, 192, 613-630, http://dx.doi.org/10.1093/gji/ggs056.

Plessix, R.-É., and Q. Cao, 2011, A parametrization study for surface seismic full waveform inversion in an acoustic vertical transversely isotropic medium: Geophysical Journal International, 185, 539556, http://dx.doi.org/10.1111/j.1365-246X.2011.04957.x.

Prieux, V., R. Brossier, Y. Gholami, S. Operto, J. Virieux, O. I. Barkved, and J. H. Kommedal, 2011, On the footprint of anisotropy on isotropic full wave-form inversion: The valhall case study: Geophysical Journal International, 187, 1495-1515, http://dx.doi.org/10.1111/j.1365246X.2011.05209.x.

Wu, Z., and T. Alkhalifah, 2015, Full waveform inversion based on scattering angle enrichment with application to real dataset: Annual International Meeting, SEG, Expanded Abstracts, 1258-1262, http://dx.doi.org/10.1190/segam2015-5922173.1.

Xu, S., D. Wang, F. Chen, G. Lambare, and Y. Zhang, 2012, Inversion on reflected seismic wave: 82nd Annual International Meeting, SEG, Expanded Abstracts, 509, 1-7.

Zhou, H., G. Zhang, and R. Bloor, 2006, An anisotropic wave equation for VTI media: 68th Annual International Conference and Exhibition, EAGE, Extended Abstracts, 68, H033. 